(RESEARCH ARTICLE)

\title{
Incidence of prostate cancer at a Referral center in the Brong Ahafo region of Ghana: A 10-year retrospective study
}

\author{
Egote Alexander Kofi ${ }^{1,}{ }^{*}$, Ossei Paul Sampane ${ }^{2}$, Ayibor William Gilbert ${ }^{3}$ and Egote Constance Amuzua 4 \\ 1 Department of Surgery (Urology Unit), Brong Ahafo Regional Hospital, Sunyani, Ghana. \\ ${ }^{2}$ Department of Pathology, School of Medicine and Dentistry, Kwame Nkrumah University of Science and Technology, \\ Kumasi. \\ ${ }^{3}$ Department of Molecular Medicine, School of Medicine and Dentistry, Kwame Nkrumah University of Science and \\ Technology, Kumasi. \\ ${ }^{4}$ Department of child health, Cape Coast Teaching Hospital, Cape Coast.
}

Publication history: Received on 06 January 2020; revised on 17 January 2020; accepted on 20 January 2020

Article DOI: https://doi.org/10.30574/wjarr.2020.5.1.0006

\begin{abstract}
Prostate cancer is gradually reaching a very high incidence in Africa, especially in the Sub-Saharan region. Understanding the dynamics in occurrence of the disorder is one approach to developing effective public health programmes and interventions that will help curb the rising incidence. This study was aimed at reducing the paucity of data on prostate cancer by assessing the incidence, patterns and presentation in the Brong Ahafo Region of Ghana. We sought to provide region-specific hardcore data that will help to assess the issue and provide remedies. All prostate disease cases recorded from the year 2009 to 2018 were retrospectively reviewed. Subjects from 40 years and above were eligible for screening. Diagnostic and screening tools for prostate cancer at the study site include family history, serum prostate specific antigen (PSA) test, digital rectal examination, urological ultrasound scan and histopathology (biopsy). Age, PSA values and year of screening/diagnosis were also retrieved from patient folders/archived reports for the study. Histological findings and parameters considered in the study included diagnosis, carcinoma grading, perineural invasion (PNI) and percentage of affected tissues (\%TA). Prostate cancer cases were 369, representing $36.4 \%$ of the 1,014 prostate diseases studied. The highest annual prevalence was recorded in 2014 with 51 cases (13.8\%). The ages of patients ranged from 46 to 101 years with a modal age range of $70-79$ years and a mean \pm SD of $72.2 \pm 9.8$. The mean PSA value recorded was $37.1 \mathrm{ng} / \mathrm{ml}( \pm 107.3)$ with predominance in the $11-20.9 \mathrm{ng} / \mathrm{ml} \mathrm{range}$. Grade group $2 \& 3$ cancers constituted the majority of prostate cancers, accounting for $149(40.4 \%)$ of valid cases. There was a weak correlation ( $p=0.091$ ) between grade of prostate cancer and perineural invasion. 38.0\% graded cancer cases had perineural invasion. There is a high incidence of prostate cancer in the Brong Ahafo Region of Ghana (32 per 100,000), predominantly advanced prostatic carcinoma. Reported cases also show high \%TA (38.7\%) and (38.0\%) PNI. Early screening for prostate diseases should be encouraged to avoid progression to malignancy and public health interventions are needed to address some of these issues.
\end{abstract}

Keywords: Prevalence; Incidence; Prostate diseases; Serum PSA; Advanced prostatic carcinoma; Ghana

\section{Introduction}

Prostate cancer incidence in Africa is on a steady rise, especially in the Sub-Saharan region [1, 2]. The dearth of proper knowledge on prostate cancer and a variety of lifestyles has accounted for the high incidence rate in Ghana [3,4]. Several documented studies have shown that countries such as Nigeria and South Africa are among the few countries with reliable and accurate statistical information on the prevalence of prostate cancer [5]. This is however not the case in Ghana as very little is known of the trend and incidence in Ghana. This therefore makes it difficult to have accurate data

\footnotetext{
${ }^{*}$ Corresponding author

E-mail address: alexanderegote@yahoo.com
} 
on prevalence and mortality rate of prostate disorders among Ghanaian men $[1,6]$. Very few studies have however documented the prevalence of prostate cancer in certain parts of the country $[3,7,8]$, with results of the studies indicating that cancer cases are on the ascendency and among them, cancer of the prostate was increasing exponentially. Region specific data is one way of providing reliable information that helps to structure a particular public health need. By examining and analysing data from our part of the country, it is our hope to provide enough evidence to support health decisions in relation to prostate cancer in the region.

\section{Methodology}

All prostate disorders recorded at the Urology Unit of the Surgical Department, Brong Ahafo Regional Hospital, SunyaniGhana from 2009 to 2018 were retrospectively reviewed. The Brong Ahafo Regional Hospital is one of the major hospitals in Ghana serving the region and beyond as it is the major referral centre in the area. The hospital has standard facility by way of infrastructure. The geographical location of the hospital, the road network of the Country and the commercial and cosmopolitan nature of the region make the hospital accessible to all the areas that share boundaries with the region and others farther away. Subjects 40 years and above (based on previous studies) were eligible for screening. Diagnostic and screening tools for prostate cancer at our facility at the time of study were family history, serum prostate specific antigen (PSA) test, digital rectal examination, urological ultrasound scan and histopathology (ultrasound guided biopsy) with 12 to 16 cores. Age, PSA values and year of screening/diagnosis were retrieved from patient folders/archives. Histological findings considered included the diagnosis, carcinoma grading, perineural invasion and percentage of affected tissues. The carcinomas were graded into grade group 1, 2\&3, 4 and 5 based on the new scoring/grading system at the time of the study. Men $<40$ years were not eligible for this study and only established prostate cancer cases were used in this study.

Data analysis was done using SPSS (Version 20.0; SPSS Inc., Chicago, IL) for descriptive statistics and correlation tests. To account for the underlying sampling frame and to provide representative population prevalence estimates, the sample population was stratified by age, year, PSA, grading and the frequency of distribution was determined among the different strata. The prevalence for the various histological presentations was also examined.

\section{Results}

During the study period, a total of 1,014 prostate diseases were investigated. Of this, there were 369 cases for prostate cancer (36.4\%) and the highest annual incidence was reported in 2014 with a prevalence rate of $13.8 \%$. This was followed in decreasing order of prevalence with the least in 2009 (3.8\%), $2010(13.0 \%), 2011(12.7 \%), 2012(12.2 \%)$, 2016(10.8\%), 2017(9.5\%), 2013(8.4\%) and 2018(7.6\%) in that order. The 369 cases selected showed a minimum and maximum of 46 and 101 years respectively. The modal age range was 70-79 years cohort with the least number of cases recorded among the $40-49$, and $\geq 100$ cohort accordingly. (Table 1)

The mean PSA value was $37.1 \mathrm{ng} / \mathrm{ml}( \pm 107.3)$ and ranged from 0.4 to $1659 \mathrm{ng} / \mathrm{ml}$. Test values fall within $11-20.9 \mathrm{ng}$ / $\mathrm{ml}(32.3 \%)$, followed by $4-10.9$ (28.9\%) and $41-50.9 \mathrm{ng} / \mathrm{ml}(10.0 \%)$. Just 6 PSA values were less than $4 \mathrm{ng} / \mathrm{ml}$ and 12 were greater than $100 \mathrm{ng} / \mathrm{m}$ (Table 1). Histologically, the new Gleason score system was used to grade 366 (99.2\%) of the prostate cancer cases. GG2 \&3 was the dominant grade representing 149 cases (40.4\%) followed by GG4 in 100 cases (27.1\%), $77(21.0 \%)$ and $40(10.8 \%)$ of the cases in GG1 and GG5 respectively. Where three of the cases were not graded (Table 1$)$. The association $(\mathrm{P}$-value $=0.091$ ) between prostate cancer rating and perineural invasion per our data was not significant. Of the 321 data on perineural invasion for the 366 graded adenocarcinomas, 122 (38.0\%) had perineural invasion and $76 \%$ of the biopsies tested at our facility had carcinoma-affected tissues within the range of $40-100 \%$. These were mainly associated with advanced form of prostate cancer. (Table 2) 
Table 1 Frequency distribution of year, age, PSA values and grading of prostate cancer.

\begin{tabular}{|c|c|c|c|c|}
\hline & Frequency & Percentage (\%) & Total $(n=369)$ & Mean (SD) \\
\hline \multicolumn{5}{|l|}{ Year } \\
\hline 2009 & 14 & 3.8 & & \\
\hline 2010 & 48 & 13.0 & & \\
\hline 2011 & 47 & 12.7 & & \\
\hline 2012 & 45 & 12.2 & & \\
\hline 2013 & 31 & 8.4 & 369 & \\
\hline 2014 & 51 & 13.8 & & \\
\hline 2015 & 30 & 8.1 & & \\
\hline 2016 & 40 & 10.8 & & \\
\hline 2017 & 35 & 9.5 & & \\
\hline 2018 & 28 & 7.6 & & \\
\hline \multicolumn{5}{|l|}{ Age } \\
\hline $40-49$ & 3 & 0.8 & & \\
\hline $50-59$ & 35 & 9.5 & & \\
\hline $60-69$ & 96 & 26.0 & 356 & $72.2(9.8)$ \\
\hline 70-79 & 135 & 36.6 & & \\
\hline $80-89$ & 72 & 19.5 & & \\
\hline $90-99$ & 14 & 3.8 & & \\
\hline$\geq 100$ & 1 & 0.3 & & \\
\hline \multicolumn{5}{|l|}{ PSA } \\
\hline$<4$ & 6 & 1.7 & & \\
\hline $4-10.9$ & 101 & 28.9 & & \\
\hline $11-20.9$ & 113 & 32.3 & & \\
\hline 21-30.9 & 30 & 8.6 & & \\
\hline $31-40.9$ & 20 & 5.7 & 350 & $37.1(107.3)$ \\
\hline $41-50.9$ & 35 & 10.0 & & \\
\hline $51-60.9$ & 9 & 2.6 & & \\
\hline $61-70.9$ & 4 & 1.1 & & \\
\hline 71-80.9 & 2 & 0.6 & & \\
\hline $81-90.9$ & 3 & 0.9 & & \\
\hline $91-100.9$ & 15 & 4.3 & & \\
\hline$>100.9$ & 12 & 3.4 & & \\
\hline \multicolumn{5}{|l|}{ Grading } \\
\hline GG1 & 77 & 21.0 & & \\
\hline GG2\&3 & 149 & 40.4 & 366 & $7.24(1.02)$ \\
\hline GG4 & 100 & 27.1 & & \\
\hline GG5 & 40 & 10.8 & & \\
\hline Ungraded & 3 & 0.5 & & \\
\hline
\end{tabular}


Egote et al. / World Journal of Advanced Research and Reviews, 2020, 05(01), 050-057

Table 2 Grading of adenocarcinoma with Perineural invasion, tissue affected and serum PSA.

\begin{tabular}{|c|c|c|c|c|c|c|}
\hline & \multicolumn{6}{|c|}{ GRADING } \\
\hline & GG1 & GG2\&3 & GG4 & GG5 & Total & p-value \\
\hline \multicolumn{7}{|l|}{ PNI } \\
\hline $\mathrm{N}$ & 38 & 86 & 56 & 19 & 199 & 0.091 \\
\hline $\mathrm{P}$ & 34 & 47 & 24 & 17 & 122 & \\
\hline \multicolumn{7}{|l|}{$\%$ TA } \\
\hline$<10$ & 2 & 8 & 2 & & 12 & \\
\hline $10-39$ & 14 & 21 & 20 & 8 & 63 & \\
\hline $40-69$ & 29 & 42 & 29 & 17 & 117 & 0.446 \\
\hline $70-100$ & 26 & 52 & 33 & 10 & 121 & \\
\hline \multicolumn{7}{|l|}{ PSA } \\
\hline$<4$ & 2 & 2 & 1 & 1 & 6 & \\
\hline $4-10.9$ & 25 & 39 & 24 & 12 & 100 & \\
\hline $11-20.9$ & 23 & 46 & 33 & 11 & 113 & \\
\hline $21-30.9$ & 8 & 13 & 4 & 5 & 30 & \\
\hline $31-40.9$ & 2 & 11 & 5 & 2 & 20 & 0.539 \\
\hline $41-50.9$ & 5 & 14 & 12 & 4 & 35 & \\
\hline $51-60.9$ & 2 & 2 & 4 & 1 & 9 & \\
\hline $61-70.9$ & & 1 & 2 & 1 & 4 & \\
\hline $71-80.9$ & 2 & & & & 2 & \\
\hline $81-90.9$ & 1 & 2 & & & 3 & \\
\hline $91-100.9$ & & 8 & 5 & & 13 & \\
\hline$>100.9$ & 2 & 6 & 4 & & 12 & \\
\hline
\end{tabular}

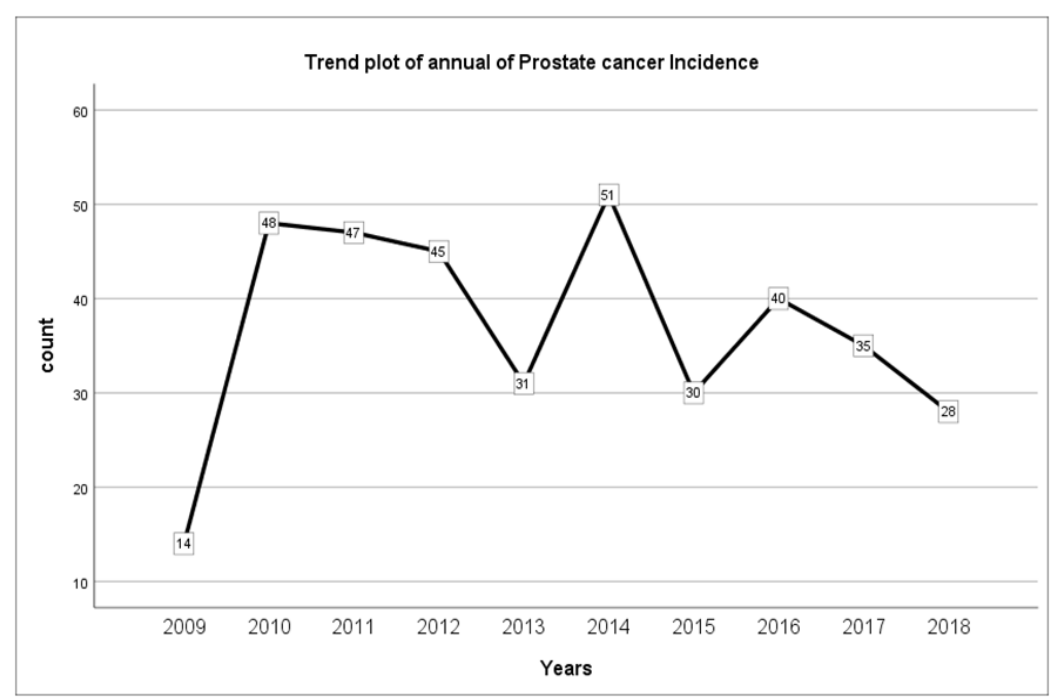

Figure 1 Yearly trend of annual incidence of Prostate cancer over the study period from 2009-2018. 


\section{Discussion}

Comparatively, Black men, particularly those of sub-Saharan or West African descent, are noted to have the highest incidence frequency of prostate cancer worldwide [1]. Therefore, it is not surprising that prostate cancer occurred in $36.4 \%$ of all prostate diseases reported at the site of the analysis. Although this rate is lower than the $40.07 \%$ recorded in our previous studies [9] and the 39.0\% prevalence recorded in Kumasi by Gyamfi et al., [10] in 2014. Similar findings have been reported in Senegal and South Africa with a prevalence rate of $30.6 \%$ and $43 \%$ respectively [2]. A contrasting prevalence rate of $10.0 \%$ and $15.7 \%$ respectively was recorded in Tobago and Nigeria studies [1].

The incidence of the disease reached its peak in 2014 with 51 cases. The year 2010 saw the second highest number of cases of prostate cancer in the regional hospital, followed by 2011 and 2012, with the lowest incidence in 2009 (Table 1; Figure 1) some important inferences can be made from the fluctuations observed in the annual incidence rates; from 14 in 2009 to 48 cases in 2010. Steadily the rate as can be seen from (Figure 1) declined to 31in 2013 followed by a sharp rise, peaking in 2014 and the subsequent decline to 28 case in 2018. These fluctuations can be due to various factors such as successful public health programs on prostate cancer that ensure that patients regularly visit our health facility center for screening and improved diagnostic accuracy. The use of serum PSA in most countries has also raised increased detection rate and concomitant increased prevalence rate $[11,12]$. Some literature findings show that the prevalence rate in Asia has decreased to 31.12\% [13], while in the Arab world the opposite has been reported [14]. The decline in prevalence rate in our study shows that although Ghanaian men are at high risk but the measures put in place are making positive impact and thus, needs to be strengthened.

Prostate cancer is prevalent among the elderly and is often branded as "elderly disease." In contrast to whites' population, African people are at increased risk as early as 40 years of age $[4,15]$. While our study findings validate this statement by reporting a minimum and maximum age of 46 and 101 years respectively, a similar prevalent situation among younger men $(<40$ years of age) is very likely to be recorded. The mean age in Iran was 66.2 years [16] whiles that of Nigeria, Kenya and Senegal recorded 68, 67 and 69 years respectively [2]. Togo and Burkina Faso also recorded a mean age of 70 and 71.5 years respectively $[16,17]$ and these are by far to the best of our knowledge, the closest to our study across the continent. It is very likely that this outcome may have compensated for the proximity of the two countries to Ghana. Given the literature examined, it can be concluded that the risk of developing the disease is higher for African men in their 60s and 70s. Although it is recognized that African men are predisposed to 40 years and above $[4,15,18,19]$, this study showed a very low prevalence among those in their 40 s $(0.8 \%)$. Early stage prostate cancer is asymptomatic until it enters the advanced stage. Therefore, this may have led to the low prevalence of men in their $40 \mathrm{~s}$. In any event, most of the cases brought to our facility are in the aggressive stage of differentiation. Studies have confirmed high mortality rates of prostate cancer in Africa, suggesting the least chance of survival, especially at advanced stages [15]. A significant relationship has been identified between tumor grading and age, and this has shown that tumors progress to more lethal forms at the very late stage of life and are therefore likely to cause elderly death compared to young adults $[8,10]$.

Since it was introduced in the diagnosis of prostate cancer, Serum PSA has attracted much criticism as a diagnostic tool. Serum PSA has been suggested to produce $70 \%$ false positives, rendering it an ineffective method for diagnosing prostate cancer [20]. According to Glady et al., [12], serum PSA has limitations which questioned its efficacy. Their report had it that serum PSA did not reliably predict tumor grade at metastatic stage. It is also not known to be a prostate cancer specific antigen, but only represents the volume of the tumor. As a consequence, many conditions such as prostatitis and benign prostate hyperplasia can affect serum PSA, resulting in false positive or negative outcomes. Yarney et al [21] also stated that more (poorly differentiated) lethal tumor stage is likely to produce less PSA serum. Our results share similar sentiments with Glady et al [12] as no statistical association between serum PSA and tumor grade has been identified (Table 2, $\mathrm{p}$-value $=0.539$ ). This agreed, however, with the statement that poorly differentiated adenocarcinoma results in less PSA serum than adequately differentiated adenocarcinomas (Table 2).

In our study, $1.7 \%$ (Table 2) of the PSA serum was below the normal value of $4.0 \mathrm{ng} / \mathrm{ml}$. The dominant PSA range was 4 to $20.9 \mathrm{ng} / \mathrm{ml}$, while $37.2 \%$ (Table 2) was > $20.9 \mathrm{ng} / \mathrm{ml}$. Yarney et al. [21] registered a contrasting trend in Accra, with 14.1 percent at $<10 \mathrm{ng} / \mathrm{ml}$ and 12.2 percent at 10 to $20 \mathrm{ng} / \mathrm{ml}$. 84.8 percent of patients receiving serum PSA above $20 \mathrm{ng} / \mathrm{ml}$ were also reported by Saadat et al., [22]. The mean PSA value of our sample (37.5 ng / ml) (Table 2) was much lower than that of Shih et al., [23] (134.4ng / ml) and Saadat et al., [22] (233.3 ng / ml) but higher than other reported values [16, 24].

Despite the backlash, serum PSA has been helpful in diagnosing asymptomatic prostate cancer [25] in other facilities and in our facility as well. This must therefore be used for accurate diagnosis in combination with urological ultrasound screening and histology. 
In this study, the Gleason score for tumors was consistent with most studies [16]. Most tumors were identified at the late stage, mainly moderately differentiated, although very few were diagnosed early. Polar results have been reported by Yarney et al., [21] and Saadat et al., [22] showing predominant well and poorly differentiated adenocarcinomas. Nevertheless, we present our results with caution, taking into account the adopted new grading system. In the context of the time and the grading system available during the course of the study, we present our findings.

Perineural invasion is the proliferation of endoneurium, perineurium, or epineurium cancer cells. This is commonly linked to advanced (metastatic) stage of tumour [22]. Among tumour grades and perineural invasion, there was no significant correlation between the two variables (Table 2 , p-value $=0.091$ ). Histologically only advanced tumours (poorly formed glands) have been shown to invade surrounding nerve cells. One hundred and twenty-two people were affected by cancer in the prostate's underlying nerve cells, 88(72.1\%) (G2-G5) of which were linked to poorly formed prostate cancer and only $34(27.9 \%$ (GG $<6$ or $\mathrm{GG}=6$ were linked to well-formed glands (Table 2). Because most people were diagnosed at an advanced stage, most of them had more than $50 \%$ of tissues affected. A higher proportion of affected tissues indicated the widespread tumour and possible metastasis to other organs at 70\%. There was, however, no substantial correlation between the proportion of TA and tumour grades (Table 2, p-value $=0.446$ ).

\section{Conclusion}

There is a high incidence of prostate cancer in the Brong Ahafo Region of Ghana (32 per 100,000), predominantly advanced prostatic carcinoma, and a prevalence rate of 36.4\%. Reported cases also show high \%TA (38.7\%) and (38.0\%) PNI. Early screening for prostate diseases should be encouraged to avoid progression to malignancy and public health interventions are needed to address some of these issues because, usually, prostate cancer is asymptomatic at the early pathogenic stage hence the diagnosis. All men $>39$ years should go for screening to ascertain conditions of their prostate. This will curtail late diagnosis and metastasis of cancer to other areas. Serum PSA should be used together with ultrasound scan and histology as a diagnostic tool to ensure precise and accurate diagnosis and management of prostate cancer.

\section{Limitations}

The study only presented results over a 10-year period from 2009 to 2018 from the Brong Ahafo region only and therefore national trends and prevalence are not known. However the study should be appreciated in its context of time frame and available resources at the facility and the fact that the Brong Ahafo region lies in the middle belt of the country and borders with both southern and northern sectors as well as the western region and data from the region is to some extent representative of the national picture.

\section{Compliance with ethical standards}

\section{Acknowledgments}

We want to thank the urology team of Brong Ahafo Regional Hospital for their cooperation and support in the process of data collection. We also want to express our gratitude to the pathology team from Kwame Nkrumah University Science and Technology for data analyzing.

\section{Disclosure of conflict of interest}

The authors declare that they have no conflict of interest.

\section{Statement of ethical approval}

Ethical approval was sought from the Committee on Human Research and Publication Ethics from our institution and was approved before the commencement of this project.

\section{Statement of informed consent}

Individual consent sought for the screening. 


\section{References}

[1] Odedina FT, Akinremi TO, Chinegwundoh F, Roberts R, Yu D, Reams RR et al. (2009). Prostate Cancer Disparities in Black Men of African Descent: A Comparative Literature Review of Prostate Cancer Burden among Black Men in the United States, Caribbean, United Kingdom, and West Africa. Infectious Agents and Cancer, 4, S2.

[2] Jalloh M, Niang L, Ndoye M, Labou I and Gueye SM. (2013). Prostate Cancer in Sub Saharan Africa. Journal of Nephrology and Urology Research, 1, 15-20.

[3] Obu R. (2014). Review: Men's Health Foundation Ghana. International Research on Medical Sciences, 2, $39-46$.

[4] Egote AK, Ossei PPS, Agyemang-Duah E, Quarshie E and Taylor J. (2018). Age as a Risk Factor for Prostate Diseases: A 6-Year Selective Prospective Study among Males in the Brong Ahafo Region of Ghana. Journal of Medical Research, 4, 154-157.

[5] Adeloye D, David RA, Aderemi AV, Iseolorunkanmi A, Oyedokun A, Iweala EE, et al. (2016). An Estimate of the Incidence of Prostate Cancer in Africa: A Systematic Review and Meta-Analysis. PLoS ONE, 11, e0153496.

[6] Egote AK, Ossei PPS, Taylor J and Bortier T. (2018). Enucleation of a Giant Prostatic Hyperplasia in Ghana: A Case Report and Mini Literature Review. Case Reports in Clinical Medicine, 7, 583-593.

[7] Arthur FKN, Yeboah FA, Adu-Frimpong M, Sedudzi EE and Boateng KA. (2005). Prostate Cancer Screening in Ghana: A Clinical Benefit? Journal of science and Technology, 25, 9-12.

[8] Laryea DO, Awuah B, Amoako YA, Osei-Bonsu E, Dogbe J and Larsen- Reindorf R. (2014). Cancer Incidence in Ghana, 2012: Evidence from Population-Based Cancer Registry. BMC Cancer, 14, 362.

[9] Egote AK, Ossei PPS, Agyeman-Duah E, Taylor J and Quarshie E. (2019). Patterns and Presentation of Prostate Cancer in the Brong Ahafo Region of Ghana: A 6-Year Single Center Retrospective Study. Health, 11(4), 351-360.

[10] Gyamfi MA, Osei OA and Osabutey CK. (2014). Efficacy of Supportive Histomorphological Features in Prostate Cancer Diagnosis. Medical \& Surgical Urology, 3, 142.

[11] Thompson IM and Ankerst DP. (2007). Prostate-specific antigen in the early detection of prostate cancer. Cmaj, 176(13), 1853-1858.

[12] Glady A, Hamidah E, Prasetyo RB and Sutiono AB. (2016). Pattern of Prostate Specific Antigen and Gleason Score in Relation to Imunohistochemistry Features in Prostate Adenocarcinoma Patients in Dr. Hasan Sadikin General Hospital. International Journal of Integrated Health Sciences, 4, 1-7.

[13] Suzuki K. (2009). Epidemiology of Prostate Cancer and Benign Prostatic Hyperplasia. JMA Journal, 52, $478-483$.

[14] Hilal L, Shahait M, Mukherji D, Charafeddine M, Farhat Z and Temraz S. (2015). Prostate Cancer in the Arab World: A View from the Inside. Clinical Genitourinary Cancer, 13, 505-511.

[15] Heyns CF, Fisher M, Lecuona A and van der Merwe A. (2011). Prostate Cancer among Different Racial Groups in the Western Cape: Presenting Features and Management. South African Medical Journal, 101, 267-270.

[16] Niroomand H, Nowroozi M, Ayati M, Jamshidian H, Arbab A and Momeni SA. (2016). Relationship between Perineural Invasion in Prostate Needle Biopsy Specimens and Pathologic Staging after Radical Prostatectomy. Nephro-Urology Monthly, 8, e36022.

[17] Amégbor K, Yao Seddoh T, Tengué K, Songne-Gnamkoulamba B, Napo-Koura G and James K. (2009). Epidemiology and Histopronostic of Prostatic Cancer in Togo: About 202 Cases Diagnosed at the Laboratory of Pathology of the Tokoin Teaching Hospital of Lome. Progrès en Urologie, 19, 112-115.

[18] Kabore FA, Zango B, Sanou A, Yameogo C and Kirakoya B. (2011). Prostate Cancer Outcome in Burkina Faso. Infectious Agents and Cancer, 6, S6.

[19] Delongchamps NB, Singh A and Haas GP. (2006). The Role of Prevalence in the Diagnosis of Prostate Cancer. Cancer Control, 13, 158-168.

[20] Luboldt HJ, Schindler JF and Rubben H. (2007). Age-Specific Reference Ranges for Prostate-Specific Antigen as a Marker for Prostate Cancer. EAU-EBU Update Series, 5, 38-48.

[21] Yarney J, Vanderpuye V and Mensah J. (2013). Clinicopathologic Features and Determinants of Gleason Score of Prostate Cancer in Ghanaian Men. Urologic Oncology: Seminars and Original Investigations, 31, 325-330. 
[22] Saadat SH, Barghoutha I, Kazzazib A, Momtahenb S, Djavanb B and Chamssuddina A. (2012). Risk Factors Associated with Perineural Invasion in Prostate Cancer. African Journal of Urology, 18, 82-86.

[23] Shih WJ, Gross K, Mitchell B, Collins J, Wierzbinski B, Magoun S, et al. (1992). Prostate Adenocarcinoma Using Gleason Scores Correlates with Prostate Specific Antigen and Prostate Acid Phosphatase Measurements. Journal of the National Medical Association, 84, 1049-1050.

[24] Vargas SO, Jiroutek M, Welch WR, Nucci MR, D'Amico AV and Renshaw AA. (1999). Perineural Invasion in Prostate Needle Biopsy Specimens Correlation with Extra prostatic Extension at Resection. American Journal of Clinical Pathology, 111, 223-228.

[25] Altuwaijri S. (2012). Role of Prostate Specific Antigen (PSA) in Pathogenesis of Prostate Cancer. Journal of Cancer Therapy, 3, 331-336.

\section{How to cite this article}

Egote AK, Ossei Paul PS, Ayibor WG and Egote CA. (2020). Incidence of prostate cancer at a Referral center in the Brong Ahafo region of Ghana: A 10-year retrospective study. World Journal of Advanced Research and Reviews, 5(1), 50-57. 